\title{
FUZZIFYING FILTERS IN LATTICE IMPLICATION ALGEBRAS
}

\author{
SeOn Yu Kim and Eun Hwan RoH*
}

\begin{abstract}
We introduce the concepts of a fuzzifying filter and a fuzzifying implicative filter in lattice implication algebras.
\end{abstract}

\section{Introduction}

In 1993, Y. Xu [9] proposed the concept of lattice implication algebras, and discussed some properties. Also, in [10], Y. Xu and K. Y. Qin introduced the notions of a filter and implicative filter in a lattice implication algebra, and investigated properties. The present authors investigated properties of lattice implication algebras $([3,6,8,11,12])$. After the introduction of the concept of fuzzy set by L. A. Zadeh [15] several researchers were conduced on the generalizations of the notion of fuzzy set. The concepts of "fuzzifying" was published by M. S. Ying [13], as elementally developed so call fuzzifying topology with the semantic method of continuous valued logic. J. Z. Shen [14] further established the theory of fuzzifying groups based on complete residuated latticevalued logic. Recently, many mathematical papers have been written investigating the fuzzifying concepts, and applying the fuzzifying concepts to various mathematics, for example, topology and algebra and so on (see $[1,2,16,17])$. In this paper, using the Ying's idea, we introduce fuzzifying filters and fuzzifying implicative filters in lattice implication algebras, and study some properties.

Received June 1, 2009. Accepted August 17, 2009.

2000 Mathematics Subject Classification. Primary 03G10, 06B10, 94D05.

Key words and phrases. Fuzzy logic, Lattice implication algebra, fuzzifying (implicative) filter.

*Corresponding author. Tel.: +82 557401232.

This paper was supported by the research fund in Chinju National University of Education, 2009. 


\section{Preliminaries}

We recall a few definitions and properties. Lattice implication algebras have been studied in details(see $[4,5,9])$.

By a lattice implication algebra we mean a bounded lattice $L=$ $(L, \vee, \wedge, 0,1)$ with order-reversing involution " ," and a binary operation " $\rightarrow$ " satisfying the following axioms for all $x, y, z \in L$ :

(I1) $x \rightarrow(y \rightarrow z)=y \rightarrow(x \rightarrow z)$,

(I2) $x \rightarrow x=1$,

(I3) $x \rightarrow y=y^{\prime} \rightarrow x^{\prime}$,

(I4) $x \rightarrow y=y \rightarrow x=1 \Rightarrow x=y$,

(I5) $(x \rightarrow y) \rightarrow y=(y \rightarrow x) \rightarrow x$,

(L1) $(x \vee y) \rightarrow z=(x \rightarrow z) \wedge(y \rightarrow z)$,

(L2) $(x \wedge y) \rightarrow z=(x \rightarrow z) \vee(y \rightarrow z)$.

We can define a partial ordering " $\leq$ " on a lattice implication algebra $L$ by $x \leq y$ if and only if $x \rightarrow y=1$.

In a lattice implication algebra $L$, the following hold: for all $x, y, z \in$ $L$,

$$
\begin{gathered}
0 \rightarrow x=1,1 \rightarrow x=x \text { and } x \rightarrow 1=1, \\
x^{\prime}=x \rightarrow 0, x=x^{\prime} \rightarrow 0, \\
x \leq y \rightarrow x \\
(x \rightarrow y) \rightarrow((y \rightarrow z) \rightarrow(x \rightarrow z))=1 .
\end{gathered}
$$

Let $F$ be a subset of a lattice implication algebra $L . F$ is called a filter of $L$ if it satisfies for all $x, y \in L$ :

(F1) $1 \in F$,

(F2) $x \in F$ and $x \rightarrow y \in F$ imply $y \in F$.

$F$ is called an implicative filter of $L$, if it satisfies (F1) and

(F3) $x \rightarrow(y \rightarrow z) \in F$ and $x \rightarrow y \in F$ imply $x \rightarrow z \in F$ for all $x, y, z \in L$.

In a broad sense, fuzzy logic is viewed as a system of concepts, principles and methods for dealing with modes of reasoning that are approximate rather than exact. There is a connection between degrees of membership in fuzzy sets and degrees of truth of fuzzy statements which is explained as follows.

Given a fuzzy set $A$, the membership degree $A(x)$ for any element $x$ in the underlying universal set $X$ may be interpreted as the degree of 
truth of the fuzzy statement " $x$ is a member of $A$ ". Conversely, given an arbitrary statement " $x$ is $F$ " where $x$ is from the set $X$ and $F$ is a fuzzy linguistic expression, its degree of truth may be interpreted as the membership degree $A(x)$ by which a fuzzy set $A$ characterized by the linguistic expression $F$ is defined in a given context.

In the beginning, Lukasiewicz study the three valued logic (the truth values are 0,1 and $\frac{1}{2}$ ). The classical definitions of the five connectives $(\wedge, \vee, \rightarrow, \leftrightarrow$ and $\neg)$ in terms of truth values 0 and 1 are preserved in three valued logic.

In second step Lukasiewicz generalized the three valued logic to what is called $n$-valued logic. The $n$ truth values (or degrees of truth) in this generalized logic are assumed to be relation numbers in the closed unit interval $[0,1]$. Allowing $n$ to go to infinity and the truth value to be any real number in $[0,1]$, which called continuous valued logics.

In continuous valued logic, the behaviors of the five logical connectives are given by the following equations:

$$
\begin{gathered}
{[\neg p]:=1-[p],} \\
{[p \wedge q]:=\min ([p],[q]),} \\
{[p \vee q]:=\max ([p],[q]),} \\
{[p \rightarrow q]:=\min (1,1-[p]+[q]),} \\
{[p \leftrightarrow q]:=[(p \rightarrow q) \wedge(q \rightarrow p)],}
\end{gathered}
$$

where $[p]$ is the degree of truth of a statement $p$.

Using the degrees of truth explained above one may deduce the following. For any fuzzy sets $\mathfrak{A}$ and $\mathfrak{B}$ of a nonempty set $X$ :

$$
\begin{gathered}
{[A \in \mathfrak{A}]:=\mathfrak{A}(A),} \\
{[\forall A(A \in \mathfrak{A})]:=\inf _{A \subseteq X} \mathfrak{A}(A),} \\
\exists A(\mathfrak{A}(A)):=\neg \forall A \neg \mathfrak{A}(A), \\
\mathfrak{A} \subseteq \mathfrak{B}:=\forall A(A \in \mathfrak{A} \rightarrow A \in \mathfrak{B}), \\
\mathfrak{A} \equiv \mathfrak{B}:=(\mathfrak{A} \subseteq \mathfrak{B}) \wedge(\mathfrak{B} \subseteq \mathfrak{A}),
\end{gathered}
$$

$$
[a]:=a \text { if } a \in[0,1] .
$$

1 .

Let $p$ be any statement. By $\vDash p$ we mean that $p$ is a tautology i.e., $[p]=$

Through this paper, the binary operation " $\rightarrow$ " in a lattice implication algebra will be denoted by juxtaposition, and for any nonempty set 
$X$ we denote $\mathcal{P}(X)$ and $\mathcal{F}(X)$ are the family of all subsets of $X$ and the fuzzy power set of $X$, respectively.

\section{Fuzzifying (implicative) filters}

Definition 3.1. Let $L$ be a lattice implication algebra and $A \in \mathcal{F}(L)$. We set

$$
\begin{aligned}
& F_{1}(A):=(\forall x)(x \in A \rightarrow 1 \in A), \\
& F_{2}(A):=(\forall x)(\forall y)((x y \in A) \wedge(x \in A) \rightarrow y \in A), \\
& F_{3}(A):=(\forall x)(\forall y)(\forall z)((x(y z) \in A) \wedge(x y \in A) \rightarrow x z \in A) .
\end{aligned}
$$

The unary fuzzy predicate $F, I F \in \mathcal{F}(\mathcal{P}(L))$ are interpreted as the fuzzy properties to be a fuzzifying filter in $L$ and to be a fuzzifying implicative filter in $L$ respectively, and are given as follows:

$$
F(A):=F_{1}(A) \wedge F_{2}(A) \text { and } I F(A):=F_{1}(A) \wedge F_{3}(A) .
$$

The following Proposition is simple, but sometimes this results is very useful.

Proposition 3.2. Let $L$ be a lattice implication algebra and $\vDash F_{1}(A)$. Then for any $x \in L$, we have

$$
[x \in A] \leq[1 \in A] .
$$

Theorem 3.3. Let $L$ be a lattice implication algebra and $\vDash F(A)$. If $x \leq y$ in $L$, then $[x \in A] \leq[y \in A]$.

Proof. Suppose that $[x \in A]>[y \in A]$. Then we have

$$
\begin{aligned}
& \inf _{x, y} \min (1,1-\min ([x y \in A],[x \in A])+[y \in A]) \\
& =\inf _{x, y} \min (1,1-\min ([1 \in A],[x \in A])+[y \in A]) \quad[\text { by }(1)] \\
& =\inf _{x, y} \min (1,1-[x \in A]+[y \in A]) \quad[\text { by Proposition 3.2] } \\
& <1 .
\end{aligned}
$$

This is a contradiction. Therefore we get $[x \in A] \leq[y \in A]$.

Theorem 3.4. Let $L$ be a lattice implication algebra. Then we have $\vDash I F \subseteq F$.

Proof. We prove that $\left[\forall A\left(A \in F_{2}\right)\right] \geq\left[\forall A\left(A \in F_{3}\right)\right]$.

$$
\begin{aligned}
& {\left[\forall A\left(A \in F_{2}\right)\right]} \\
& =\inf _{A} \inf _{x, y} \min (1,1-\min ([x y \in A],[x \in A])+[y \in A]) \\
& =\inf _{A} \inf _{x, y} \min (1,1-\min ([1(x y) \in A],[1 x \in A])+[1 y \in A]) \\
& \geq \inf _{A} \inf _{x, y, z} \min (1,1-\min ([z(x y) \in A],[z x \in A])+[z y \in A]) \\
& =\left[\forall A\left(A \in F_{3}\right)\right] .
\end{aligned}
$$


Remark 3.5. In fuzzifying setting the statement $\vDash F \subseteq I F$ may not be true as illustrated by the following example.

Example 3.6. Let $L:=\{0, a, b, c, 1\}$. Define the partially ordered relation on $L$ as $0<a<b<c<1$, and define $x \wedge y:=\min \{x, y\}$, $x \vee y:=\max \{x, y\}$ for all $x, y \in L$ and "" and " $\rightarrow$ " (also we will use the juxtaposition) as follows:

\begin{tabular}{c|c}
$x$ & $x^{\prime}$ \\
\hline 0 & 1 \\
$a$ & $c$ \\
$b$ & $b$ \\
$c$ & $a$ \\
1 & 0
\end{tabular}

\begin{tabular}{c|ccccc} 
& 0 & $a$ & $b$ & $c$ & 1 \\
\hline 0 & 1 & 1 & 1 & 1 & 1 \\
$a$ & $c$ & 1 & 1 & 1 & 1 \\
$b$ & $b$ & $b$ & 1 & 1 & 1 \\
$c$ & $a$ & $b$ & $b$ & 1 & 1 \\
1 & 0 & $a$ & $b$ & $c$ & 1
\end{tabular}

Then $L$ is a lattice implication algebra. Define the degree of truth of the fuzzy statement " $x$ is a member of $A$ " by

$$
A(x):=\left\{\begin{array}{cc}
0.8 & \text { if } x=1 \\
0.6 & \text { if } x=c \\
0 & \text { otherwise }
\end{array}\right.
$$

for all $x \in L$. Then it is easy check that $A$ is a fuzzifying filter of $L$, but $A$ is not a fuzzifying implicative filter of $L$ since

$$
\min (1,1-\min ([a(b 0) \in A],[a b \in A])+[a 0 \in A])=0.8 .
$$

Therefore we get $\not \models F \subseteq I F$.

Theorem 3.7. Let $L$ be a lattice implication algebra. Then for any $A_{\lambda}(\lambda \in \Lambda) \in \mathcal{F}(L)$, we have

$$
\vDash(\forall \lambda)\left((\lambda \in \Lambda) \rightarrow I F\left(A_{\lambda}\right)\right) \rightarrow I F\left(\underset{\lambda \in \Lambda}{\cap} A_{\lambda}\right) .
$$

Proof.

$$
\begin{aligned}
{\left[F_{1}\left(\cap_{\lambda \in \Lambda} A_{\lambda}\right)\right] } & =\inf _{x} \min \left(1,1-\left[x \in \cap_{\lambda \in \Lambda} A_{\lambda}\right]+\left[1 \in \cap_{\lambda \in \Lambda} A_{\lambda}\right]\right) \\
& =\inf _{x} \min \left(1,1-\inf _{\lambda \in \Lambda}\left[x \in A_{\lambda}\right]+\inf _{\lambda \in \Lambda}\left[1 \in A_{\lambda}\right]\right) \\
& \geq \inf _{x} \inf _{\lambda \in \Lambda} \min \left(1,1-\left[x \in A_{\lambda}\right]+\left[1 \in A_{\lambda}\right]\right) \\
& =\inf _{\lambda \in \Lambda}\left[F_{1}\left(A_{\lambda}\right)\right], \text { and }
\end{aligned}
$$




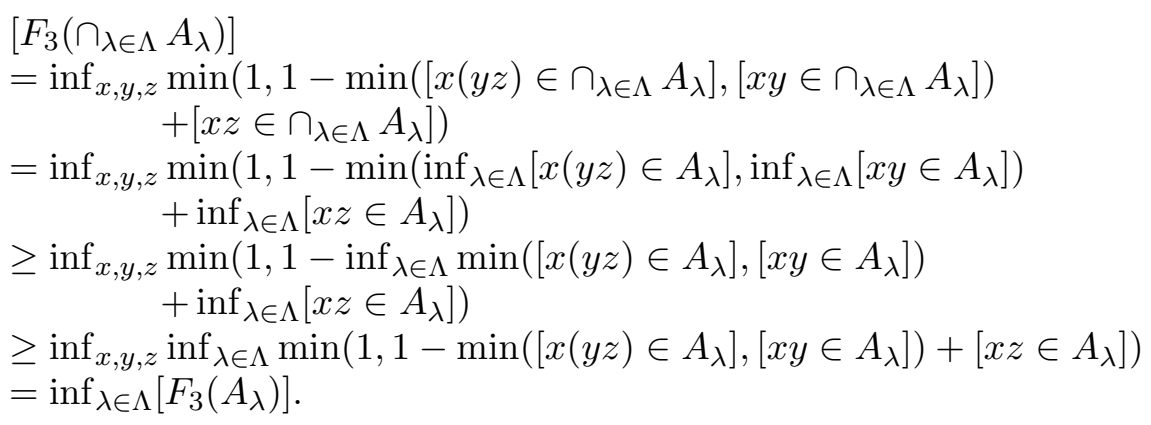

Therefore we have

$$
\begin{aligned}
{\left[\operatorname{IF}\left(\cap_{\lambda \in \Lambda} A_{\lambda}\right)\right] } & =\min \left(\left[F_{1}\left(\cap_{\lambda \in \Lambda} A_{\lambda}\right)\right],\left[F_{3}\left(\cap_{\lambda \in \Lambda} A_{\lambda}\right)\right]\right) \\
& \geq \inf _{\lambda \in \Lambda} \min \left(\left[F_{1}\left(A_{\lambda}\right)\right],\left[F_{3}\left(A_{\lambda}\right)\right]\right) \\
& =\inf _{\lambda \in \Lambda}\left[\operatorname{IF}\left(A_{\lambda}\right)\right] .
\end{aligned}
$$

By Theorems 3.4 and 3.7, we have the following result.

Corollary 3.8. Let $L$ be a lattice implication algebra. Then for any $A_{\lambda}(\lambda \in \Lambda) \in \mathcal{F}(L)$, we have

$$
\vDash(\forall \lambda)\left((\lambda \in \Lambda) \rightarrow F\left(A_{\lambda}\right)\right) \rightarrow F\left(\underset{\lambda \in \Lambda}{\cap} A_{\lambda}\right) .
$$

Theorem 3.9. Let $L$ be a lattice implication algebra and $\vDash F(A)$. Then we have

$$
\vDash A \in I F \rightarrow(\forall x)(\forall y)(x(x y) \in A \rightarrow x y \in A) .
$$

Proof.

$$
\begin{aligned}
& \inf _{x, y} \min (1,1-[x(x y) \in A]+[x y \in A]) \\
& =\inf _{x, y} \min (1,1-\min ([x(x y) \in A],[x x \in A]) \\
& \quad+[x y \in A]) \quad \quad \quad[\text { by Proposition 3.2] } \\
& \geq \inf _{x, y, z} \min (1,1-\min ([x(y z) \in A],[x y \in A])+[x z \in A]) \\
& =\left[A \in F_{3}\right] .
\end{aligned}
$$

Theorem 3.10. Let $L$ be a lattice implication algebra and $a \in L$. Then we have

$$
\vDash A \in I F \rightarrow A_{a} \in F,
$$

where $A_{a}:=\{t \in L \mid A(a t)=1\}$. 
Proof.

$$
\begin{aligned}
{\left[A_{a} \in F_{1}\right] } & =\inf _{x} \min \left(1,1-\left[x \in A_{a}\right]+\left[1 \in A_{a}\right]\right) \\
& =\inf _{x} \min \left(1,1-\left[x \in A_{a}\right]+[a 1 \in A]\right) \\
& =\inf _{x} \min (1,1-[a x \in A]+[1 \in A]) \\
& =1, \quad[\text { by Proposition 3.2] }
\end{aligned}
$$

and

$$
\begin{aligned}
& {\left[A_{a} \in F_{2}\right]} \\
& =\inf _{x, y} \min \left(1,1-\min \left(\left[x y \in A_{a}\right],\left[x \in A_{a}\right]\right)+\left[y \in A_{a}\right]\right) \\
& =\inf _{x, y} \min (1,1-\min ([a(x y) \in A],[a x \in A])+[a y \in A]) \\
& =\left[A \in F_{3}\right]
\end{aligned}
$$

Thus we have $\vDash A \in I F \rightarrow A_{a} \in F$.

\section{Cartesian product of fuzzifying implicative filters}

Definition 4.1. Let $L$ be a lattice implication algebra, $A \in \mathcal{F}(L \times L)$ and $B \in \mathcal{F}(L)$. A binary fuzzy predicate $R_{B} \in \mathcal{F}(\mathcal{P}(L \times L))$, called fuzzifying relation on $B$, is given as follows:

$$
A \in R_{B}:=(\forall x)(\forall y)((x, y) \in A \rightarrow(x \in B) \wedge(y \in B)) .
$$

Lemma 4.2. Let $L$ be a lattice implication algebra and $A, B \in \mathcal{F}(L)$. Then

$$
\vDash(A \times B) \in R_{L} \leftrightarrow(\forall x)(\forall y)((x, y) \in A \times B \leftrightarrow(x \in B) \wedge(y \in B))
$$

Theorem 4.3. Let $L$ be a lattice implication algebra and $A, B \in$ $\mathcal{F}(L)$. Then we have

$$
\vDash(A \in I F) \wedge(B \in I F) \rightarrow(A \times B \in I F) .
$$


Proof.

$$
\begin{aligned}
& {\left[F_{1}(A \times B)\right]} \\
& =\inf _{x, y} \min (1,1-[(x, y) \in A \times B]+[(1,1) \in A \times B]) \\
& =\inf _{x, y} \min (1,1-\min ([x \in A],[y \in B])+\min ([1 \in A],[1 \in B])) \\
& \geq \min \left(\inf _{x} \min (1,1-[x \in A]+[1 \in A]),\right. \\
& \left.\inf _{y} \min (1,1-[y \in B]+[1 \in B])\right) \\
& =\left[F_{1}(A) \wedge F_{1}(B)\right] \text {, and } \\
& {\left[F_{3}(A \times B)\right]} \\
& =\inf _{x_{1}, y_{1}, z_{1}, x_{2}, y_{2}, z_{2}} \min \left(1,1-\min \left(\left[\left(x_{1}, x_{2}\right)\left(\left(y_{1}, y_{2}\right)\left(z_{1}, z_{2}\right)\right) \in A \times B\right]\right. \text {, }\right. \\
& \left.\left.\left[\left(x_{1}, x_{2}\right)\left(y_{1}, y_{2}\right) \in A \times B\right]\right)+\left[\left(x_{1}, x_{2}\right)\left(z_{1}, z_{2}\right) \in A \times B\right]\right) \\
& =\inf _{x_{1}, y_{1}, z_{1}, x_{2}, y_{2}, z_{2}} \min \left(1,1-\min \left(\left[\left(x_{1}\left(y_{1} z_{1}\right), x_{2}\left(y_{2} z_{2}\right)\right) \in A \times B\right]\right. \text {, }\right. \\
& \left.\left.\left[\left(x_{1} y_{1}, x_{2} y_{2}\right) \in A \times B\right]\right)+\left[\left(x_{1} z_{1}, x_{2} z_{2}\right) \in A \times B\right]\right) \\
& =\inf _{x_{1}, y_{1}, z_{1}, x_{2}, y_{2}, z_{2}} \min \left(1,1-\min \left(\min \left(\left[x_{1}\left(y_{1} z_{1}\right) \in A\right],\left[x_{2}\left(y_{2} z_{2}\right) \in B\right]\right)\right. \text {, }\right. \\
& \left.\left.\min \left(\left[x_{1} y_{1} \in A\right],\left[x_{2} y_{2} \in B\right]\right)\right)+\min \left(\left[x_{1} z_{1} \in A\right],\left[x_{2} z_{2} \in B\right]\right)\right) \\
& \geq \min \left(\operatorname { i n f } _ { x _ { 1 } , y _ { 1 } , z _ { 1 } } \operatorname { m i n } \left(1,1-\min \left(\left[x_{1}\left(y_{1} z_{1}\right) \in A\right],\left[x_{1} y_{1} \in A\right]\right)\right.\right. \\
& \left.+\left[x_{1} z_{1} \in A\right]\right),\left(\operatorname { i n f } _ { x _ { 2 } , y _ { 2 } , z _ { 2 } } \operatorname { m i n } \left(1,1-\min \left(\left[x_{2}\left(y_{2} z_{2}\right) \in A\right],\right.\right.\right. \\
& \left.\left.\left[x_{2} y_{2} \in A\right]\right)+\left[x_{2} z_{2} \in A\right]\right) \\
& =\left[F_{3}(A) \wedge F_{3}(B)\right] \text {. }
\end{aligned}
$$

Therefore we have $[I F(A) \wedge I F(B)] \leq[I F(A \times B)]$.

Theorem 4.4. Let $L$ be a lattice implication algebra and $A, B \in$ $\mathcal{F}(L)$. Then we have

(a) $\vDash(A \times B \in I F) \rightarrow(\forall x)((x \in A \rightarrow 1 \in A) \vee(x \in B \rightarrow 1 \in B))$.

(b) If $\vDash(\forall x)((x \in A \rightarrow 1 \in B)$, then $\vDash A \times B \in I F \rightarrow B \in I F$.

Proof. (a) Since $[\operatorname{IF}(A \times B)] \leq\left[F_{1}(A \times B)\right]$, we only need to prove that $\left[F_{1}(A \times B)\right] \leq \inf _{x} \max (\min (1,1-[x \in A]+[1 \in A]), \min (1,1-[x \in$ $B]+[1 \in B]))$. Suppose that $\left[F_{1}(A \times B)\right] \geq t$ for $t \in[0,1)$. If there exists a $x \in L$ and a $y \in L$ such that $\min (1,1-[x \in A]+[1 \in A])<t$ and $\min (1,1-[x \in B]+[1 \in B])<t$ respectively, then there exists a $(x, y) \in$ $L \times L$ such that $[x \in A]>1-t+[1 \in A]$ and $[x \in B]>1-t+[1 \in B]$. 
Thus we have

$$
\begin{aligned}
(A \times B)(x, y) & =\min ([x \in A],[y \in B]) \\
& >\min (1-t+[1 \in A], 1-t+[1 \in B]) \\
& =1-t+\min ([1 \in A],[1 \in B]) \\
& =1-t+(A \times B)(1,1) .
\end{aligned}
$$

On the other hand, such a some $(x, y) \in L \times L$, we obtain

$$
\begin{aligned}
& \min (1,1-[(x, y) \in A \times B]+[(1,1) \in A \times B]) \\
& \leq 1-[(x, y) \in A \times B]+[(1,1) \in A \times B] \\
& <1-(1-t+[(1,1) \in A \times B])+[(1,1) \in A \times B] \\
& =t
\end{aligned}
$$

it is a contradiction.

(b) By (a), we can suppose that $\max \left(\inf _{x} \min (1,1-[x \in A]+[1 \in\right.$ $\left.A]), \inf _{x} \min (1,1-[x \in B]+[1 \in B])\right)=\inf _{x} \min (1,1-[x \in A]+[1 \in A])$. Then $[\operatorname{IF}(A \times B)] \leq[(\forall x)(x \in A \rightarrow 1 \in A)]=\left[F_{1}(A)\right]$. If $\vDash(\forall x)((x \in$ $A \rightarrow 1 \in B)$, we have $[x \in A] \leq[1 \in B]$ for any $x \in L$. Thus we have

$$
\begin{aligned}
& {[I F(A \times B)] \leq\left[F_{3}(A \times B)\right]} \\
& =\inf _{x_{1}, y_{1}, z_{1}, x_{2}, y_{2}, z_{2}} \min \left(1,1-\min \left(\left[\left(x_{1}, x_{2}\right)\left(\left(y_{1}, y_{2}\right)\left(z_{1}, z_{2}\right)\right) \in A \times B\right],\right.\right. \\
& \\
& \left.\left.\quad\left[\left(x_{1}, x_{2}\right)\left(y_{1}, y_{2}\right) \in A \times B\right]\right)+\left[\left(x_{1}, x_{2}\right)\left(z_{1}, z_{2}\right) \in A \times B\right]\right) \\
& =\inf _{x_{1}, y_{1}, z_{1}, x_{2}, y_{2}, z_{2}} \min \left(1,1-\min \left(\left[\left(x_{1}\left(y_{1} z_{1}\right), x_{2}\left(y_{2} z_{2}\right)\right) \in A \times B\right],\right.\right. \\
& \left.\left.\quad\left[\left(x_{1} y_{1}, x_{2} y_{2}\right) \in A \times B\right]\right)+\left[\left(x_{1} z_{1}, x_{2} z_{2}\right) \in A \times B\right]\right) \\
& \leq \inf _{x_{1}, y_{1}, z_{1}} \min \left(1,1-\min \left(\left[\left(x_{1}\left(y_{1} z_{1}\right), 1\right) \in A \times B\right],\right.\right. \\
& \left.\left.\quad\left[\left(x_{1} y_{1}, 1\right) \in A \times B\right]\right)+\left[\left(x_{1} z_{1}, 1\right) \in A \times B\right]\right) \\
& =\inf _{x_{1}, y_{1}, z_{1}} \min \left(1,1-\min \left(\min \left(\left[x_{1}\left(y_{1} z_{1}\right) \in A\right],[1 \in B]\right),\right.\right. \\
& \left.\left.\quad \min \left(\left[x_{1} y_{1} \in A\right],[1 \in B]\right)\right)+\min \left(\left[x_{1} z_{1} \in A\right],[1 \in B]\right)\right) \\
& =\inf _{x_{1}, y_{1}, z_{1}} \min \left(1,1-\min \left(\left[x_{1}\left(y_{1} z_{1}\right) \in A\right],\left[x_{1} y_{1} \in A\right]\right)+\left[x_{1} z_{1} \in A\right]\right) \\
& =\left[F_{3}(A)\right] .
\end{aligned}
$$

Therefore we get $[I F(A \times B)] \leq \min \left(\left[F_{1}(A)\right],\left[F_{3}(A)\right]\right)=[I F(A)]$.

Theorem 4.5. Let $L$ be a lattice implication algebra and $B \in \mathcal{F}(L)$. If $B^{2} \in(L \times L)$ which is defined as follows: for all $x, y \in L$,

$$
(x, y) \in B^{2}:=(x \in B) \wedge(y \in B) .
$$

Then $\vDash B \in I F \leftrightarrow B^{2} \in I F$. 
Proof. First we prove that $\vDash B \in I F \rightarrow B^{2} \in I F .\left[F_{1}\left(B^{2}\right)\right]=$ $\inf _{x, y} \min \left(1,1-\left[(x, y) \in B^{2}\right]+\left[(1,1) \in B^{2}\right]\right)=\inf _{x, y} \min (1,1-\min ([x \in$ $B],[y \in B])+[1 \in B]) \geq \inf _{x, y} \min (1,1-[x \in B]+[1 \in B])=$ $\inf _{x} \min (1,1-[x \in B]+[1 \in B])=\left[F_{1}(B)\right]$.

$$
\begin{aligned}
& {\left[F_{3}\left(B^{2}\right)\right]} \\
& =\inf _{x_{1}, y_{1}, z_{1}, x_{2}, y_{2}, z_{2}} \min \left(1,1-\min \left(\left[\left(x_{1}, x_{2}\right)\left(\left(y_{1}, y_{2}\right)\left(z_{1}, z_{2}\right)\right) \in B^{2}\right],\right.\right. \\
& \left.\left.\quad\left[\left(x_{1}, x_{2}\right)\left(y_{1}, y_{2}\right) \in B^{2}\right]\right)+\left[\left(x_{1}, x_{2}\right)\left(z_{1}, z_{2}\right) \in B^{2}\right]\right) \\
& =\inf _{x_{1}, y_{1}, z_{1}, x_{2}, y_{2}, z_{2}} \min \left(1,1-\min \left(\left[\left(x_{1}\left(y_{1} z_{1}\right), x_{2}\left(y_{2} z_{2}\right)\right) \in B^{2}\right],\right.\right. \\
& \left.\left.\quad\left[\left(x_{1} y_{1}, x_{2} y_{2}\right) \in B^{2}\right]\right)+\left[\left(x_{1} z_{1}, x_{2} z_{2}\right) \in B^{2}\right]\right) \\
& =\quad \inf _{x_{1}, y_{1}, z_{1}, x_{2}, y_{2}, z_{2}} \min \left(1,1-\min \left(\min \left(\left[x_{1}\left(y_{1} z_{1}\right) \in B\right],\left[x_{1} y_{1} \in B\right]\right),\right.\right. \\
& \left.\quad \min \left(\left[x_{2}\left(y_{2} z_{2}\right) \in B\right],\left[x_{2} y_{2} \in B\right]\right)\right) \\
& \left.\quad+\min \left(\left[x_{1} z_{1} \in B\right],\left[x_{2} z_{2} \in B\right]\right)\right) \\
& \geq \inf _{x, y, z} \min (1,1-\min ([x(y z) \in B],[x y \in B])+[x z \in B]) \\
& =\left[F_{3}(B)\right] .
\end{aligned}
$$

Conversely, $\left[F_{1}\left(B^{2}\right)\right]=\inf _{x, y} \min \left(1,1-\left[(x, y) \in B^{2}\right]+\left[(1,1) \in B^{2}\right]\right) \leq$ $\inf _{x} \min \left(1,1-\left[(x, x) \in B^{2}\right]+\left[(1,1) \in B^{2}\right]\right)=\inf _{x} \min (1,1-[x \in B]+[1 \in$ $B])=\left[F_{1}(B)\right]$

$$
\begin{aligned}
& {\left[F_{3}\left(B^{2}\right)\right]} \\
& =\inf _{x_{1}, y_{1}, z_{1}, x_{2}, y_{2}, z_{2}} \min \left(1,1-\min \left(\left[\left(x_{1}, x_{2}\right)\left(\left(y_{1}, y_{2}\right)\left(z_{1}, z_{2}\right)\right) \in B^{2}\right],\right.\right. \\
& \left.\left.\quad\left[\left(x_{1}, x_{2}\right)\left(y_{1}, y_{2}\right) \in B^{2}\right]\right)+\left[\left(x_{1}, x_{2}\right)\left(z_{1}, z_{2}\right) \in B^{2}\right]\right) \\
& =\inf _{x_{1}, y_{1}, z_{1}, x_{2}, y_{2}, z_{2}} \min \left(1,1-\min \left(\left[\left(x_{1}\left(y_{1} z_{1}\right), x_{2}\left(y_{2} z_{2}\right)\right) \in B^{2}\right],\right.\right. \\
& \left.\left.\quad\left[\left(x_{1} y_{1}, x_{2} y_{2}\right) \in B^{2}\right]\right)+\left[\left(x_{1} z_{1}, x_{2} z_{2}\right) \in B^{2}\right]\right) \\
& =\quad \inf _{x_{1}, y_{1}, z_{1}, x_{2}, y_{2}, z_{2}} \min \left(1,1-\min \left(\min \left(\left[x_{1}\left(y_{1} z_{1}\right) \in B\right],\left[x_{2}\left(y_{2} z_{2}\right) \in B\right]\right),\right.\right. \\
& \left.\left.\quad \min \left(\left[x_{1} y_{1} \in B\right],\left[x_{2} y_{2} \in B\right]\right)\right)+\min \left(\left[x_{1} z_{1} \in B\right],\left[x_{2} z_{2} \in B\right]\right)\right) \\
& \leq \inf _{x, y, z} \min (1,1-\min (\min ([x(y z) \in B],[1 \in B]), \min ([x y \in B], \\
& \leq \inf _{x, y, z} \min (1,1-\min ([x(y z) \in B],[x y \in B])+[x z \in B]) \\
& =\left[F_{3}(B)\right] .
\end{aligned}
$$




\section{References}

[1] K. M. Abd El-Hakeim and F. M. Zeyada, Some bifuzzy topologies induced by a fuzzifying topology: Part (I): Fundamental Concepts, J. Fuzzy Math. 8(4) (2000), 845-856.

[2] H. M. Ghanim, F. A. Ibrahim and M. A. Sakr, On fuzzifying filters, grills and basic proximities, J. Fuzzy Math. 8(1) (2000), 79-87.

[3] S. Y. Kim, E. H. Roh and Y. B. Jun, On ultrafilters of lattice implication algebras, Scientiae Mathematicae 2 (1998), 201-204.

[4] J. Liu and Y. Xu, Filters and structure of lattice implication algebra, Chinese Science Bulletin 42(18) (1997), 1517-1520.

[5] J. Liu and Y. Xu, On prime filters and decomposition theorem of lattice implication algebras, J. Fuzzy Math. 6(4) (1998), 1001-1008.

[6] E. H. Roh, S. Y. Kim, Y. Xu and Y. B. Jun ,Some operations on lattice implication algebras, International J. of Mathematics and Mathematical Sciences 27(1) (2001), 45-52.

[7] J. B. Rosser and A. R. Turquette, Many-Valued Logics, North-Holland, Amsterdam-New York, 1952.

[8] E. H. Roh, Y. Xu and K. Y. Qin, On implicative filters of lattice implication algebras, Submitted.

[9] Y. Xu, Lattice implication algebras, J. of Southwest Jiaotong Univ. 28(1) (1993), 20-27. (Chinese)

[10] Y. Xu and K. Y. Qin, On filters of lattice implication algebras, J. Fuzzy Math. 1 (1993), 251-260.

[11] Y. Xu. K. Y. Qin and E. H. Roh, On prime filters of lattice H implication algebras, J. Fuzzy Math. 9(3) (2001), 583-588.

[12] Y. Xu. E. H. Roh and K. Y. Qin On quotient lattice implication algebras induced by fuzzy filters, J. Fuzzy Math. 9(3) (2001), 611-617.

[13] Ming Sheng Ying, A new approach for fuzzy topology I, Fuzzy Sets and Systems 39 (1991), 303-321.

[14] J. Z. Shen, Fuzzifying groups based on complete residuated lattice-valued logic, Information Sciences 75 (1993) 165-186.

[15] L. A. Zadeh, Fuzzy sets, Inform. Control. 8 (1965), 338-353.

[16] G. J. Zhang, Semi-open sets and semi-continuity in fuzzifying topological spaces, J. Fuzzy Math. 8(4) (2000), 981-987.

[17] X. F. Zou and J. Z. Shen, Fuzzifying codes and fuzzifying prefix codes, J. Fuzzy Math. 8(2) (2000), 307-313.

Seon Yu Kim and Eun Hwan Roh

Department of Mathematics Education

and Institute of Mathematics Education, Chinju National University of Education, Junju 660-756, Korea

e-mail: sykim@cue.ac.kr

e-mail: ehroh@cue.ac.kr or ehroh0923@gmail.com 